\title{
A Novel Integrated Learning Model for Rainfall Prediction CEEMD- FCMSE -Stacking
}

\section{Xianqi Zhang}

North China University of Water Resources and Electric Power

Kai Wang ( $\boldsymbol{\nabla}$ 2416932728@qq.com )

North China University of Water Resources and Electric Power

\section{Tao Wang}

North China University of Water Resources and Electric Power

\section{Research Article}

Keywords: Rainfall Forecasting, CEEMD, RCMSE, Stacking Integrated Learning, Xixia

Posted Date: January 14th, 2022

DOI: https://doi.org/10.21203/rs.3.rs-1006290/v1

License: (c) (1) This work is licensed under a Creative Commons Attribution 4.0 International License. Read Full License 


\section{A novel integrated learning model for rainfall prediction CEEMD- FCMSE}

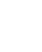

(

4

${ }^{a}$ Water Conservancy College, North china University of Water Resources and Electric Power, Zhengzhou 450046, China

${ }^{b}$ Collaborative Innovation Center of Water Resources Efficient Utilization and Protection Engineering, Zhengzhou 450046, China

${ }^{c}$ Technology Research Center of Water Conservancy and Marine Traffic Engineering, Henan Province, Zhengzhou 450046, China

*Corresponding authors: Kai Wang. Email: 2416932728@qq.com;phone: +86-152$9042-1442$

\section{Authors:}

Xianqi Zhang. E-mail: zxqi@163.com; phone: +86-158-3719-7937

Kai Wang. Email: 2416932728@qq.com; phone:+86-152-9042-1442

Tao Wang. E-mail: 1124149584@qq.com; phone: +86-157-3834-7223

Abstract: Scientific prediction of precipitation changes has important guiding value and significance for revealing regional spatial and temporal patterns of precipitation changes, flood climate prediction, etc. Based on the fact that CEEMD can effectively overcome the interference of modal aliasing and white noise, fine composite multi-scale entropy can reorganize the same FCMSE value to reduce the modal component and improve the computational efficiency, and Stacking ensemble learning can effectively and conveniently improve the fitting effect of machine learning, a rainfall prediction method based on CEEMD-fine composite multi-scale entropy and Stacking ensemble learning is constructed, and it is applied to the prediction of monthly precipitation in 
the Xixia. The results show that, under the same conditions, the CEEMD-RCMSEStacking model reduces the root mean square error by $83.48 \%$ and $62.08 \%$, and the mean absolute error by $83.25 \%$ and $61.84 \%$, respectively, compared with the single Stacking model and CEEMD-LSTM, while the goodness-of-fit coefficients improve by $15.94 \%$ and $2.34 \%$, respectively, which means that the CEEMD-RCMSE-Stacking model has higher prediction performance. The CEEMD-RCMSE-Stacking model has higher prediction performance.

Keywords: Rainfall Forecasting, CEEMD, RCMSE, Stacking Integrated Learning, Xixia

\section{Introduction}

The contradiction between water supply and demand is a major problem facing China's social development at present, while precipitation is an important way of recharging regional water resources, and long-term precipitation forecasts play an important role in the rational allocation and use of water resources (Qing et al. 2019). Precipitation is influenced by a variety of uncertainties and its process is very complex. Most of the precipitation series are non-stationary series, which not only have the characteristics of trend and periodicity, but also have randomness, sudden changes and "multi-time scale" structure, which makes the precipitation prediction accuracy relatively low. This makes the accuracy of precipitation forecasts relatively low. In recent years, scholars at home and abroad have conducted a lot of research on precipitation forecasting and have achieved fruitful results. Bidroha et al. (2020) A downscaling method based on support vector regression (SVR) to downscale rainfall at several locations in a study area to obtain future rainfall forecasts, and the performance of the SVR method is compared with downscaling methods based on correlation vector 
machines and deep learning to demonstrate the effectiveness of the method. Yu et al.(2018) explored short-long time variation information in the original rainfall time

51 series, where Support Vector Regression (SVR) was used for short-period component prediction and Artificial Neural Networks (ANN) for long-period component prediction, with results showing superior performance to traditional methods. Fethi et al. (2018) Prediction of rainfall using data retrieved from the Meteosat Second Generation (MSG) Rotationally Enhanced Visible and Infrared Imager (SEVIRI) based on the Random Forest (RF) algorithm, showing that the rainfall estimated by the scheme correlates well with that observed by rain gauges. Khan et al. (2020) The analysis was carried out using 30 years of rainfall data from 1986 - 2016 in the Langat River Basin, Malaysia. Discrete wavelet transform decomposition of the calculated drought time series and prediction of high frequency subseries using artificial neural networks. Kavya et al. (2020) An adaptive empirical model decomposition-artificial neural network model is proposed for predicting summer rainfall in India. Liu et al. (2021) Integration of improved KNearest Neighbour, Remote Sensing and Geographic Information Systems to analyse extreme rainfall data from tourist sites. Sun et al. (2021) The possibility of using the RF-SVR statistical downscaling model for extreme rainfall simulations during the flood season was explored, and the downscaling effects of the RF-SVR statistical downscaling model were compared with those of the SVR model. The results show that the deviations of daily rainfall in the Luan River basin simulated by the RF-SVR model are significantly reduced, and the prediction of extreme rainfall in the basin can be improved. Guo et al. (2010) The ANN-based statistical downscaling method is studied

71 and explored to establish the statistical relationship between large-scale climate observations and measured precipitation through ANN and apply it to study the precipitation changes in the Han River basin under future climate scenarios. Lin et al. 
74 (2021) The integrated models such as bagging integrated model and stacking integrated model were established respectively to improve the effect of short time runoff forecasting in the Andun water basin, and the results show that its stacking integrated model has a better effect on the prediction of small flow incoming water. Scholars at home and abroad have investigated the use of combinatorial methods to optimise model parameters or modify parts of the code to improve the accuracy of rainfall prediction models, however there is less research on integrated learning model methods using each single prediction model. The Stacking algorithm brings together the strengths of different models and allows the raw data to be analysed from multiple perspectives, resulting in better predictive performance. In this paper, an adaptive noise-complete ensemble empirical modal decomposition (CEEMD) method is first introduced to decompose the raw precipitation series and calculate the fine composite multiscale entropy (RCMSE) of each decomposition component. The sequences of components with similar entropy values are then reorganised into new sequences to reduce model complexity and improve computational efficiency. In the prediction stage, the reconstructed series are based on the popular KNN, RF, SVR and ANN algorithms mentioned above, and the Stacking algorithm is used to combine the advantages of the four models to analyse the raw data from multiple perspectives, thus improving the model prediction performance and applying it to the monthly precipitation prediction at the Xixia station.

\section{Research methods}

\subsection{CEEMD algorithm}

The CEEMD algorithm adaptively adds white noise to the decomposition process to solve the modal mixing problem that occurs with EMD, and to overcome the low 


$$
r_{k}(t)=r_{k-1}(t)-I M F_{k}(t)
$$

$$
I M F_{1}(t)=\frac{1}{n} \sum_{i=1}^{n} I M F_{1}^{\mathrm{i}}(t)
$$

$$
\text { Calculate the first residual signal } r_{1}(t)
$$

$$
r_{1}(t)=y(t)-I M F_{1}(t)
$$

(3) The EMD algorithm is used to decompose the signal $r_{1}(t)+\varepsilon_{1} E_{1}\left(\omega^{i}(t)\right)$ to obtain the second modal component $I M F_{2}(t)$

$$
I M F_{2}(t)=\frac{1}{n} \sum_{i=1}^{n} E_{1}\left(r_{1}(t)+\varepsilon_{1} E_{1}\left(\omega^{i}(t)\right)\right)
$$

$$
I M F_{k+1}(t)=\frac{1}{n} \sum_{i=1}^{n} E_{1}\left(r_{k}(t)+\varepsilon_{k} E_{k}\left(\omega^{i}(t)\right)\right)
$$


120 decomposed, resulting in $\mathrm{K}$ modal components. The decomposed final residual signal

121 is

$$
R(t)=y(t)-\sum_{i=1}^{K} I M F_{k}(t)
$$

The original rainfall signal is therefore decomposed into

$$
y(t)=\sum_{i=1}^{K} I M F_{k}(t)+R(t)
$$

\subsection{Fine-composite multiscale entropy}

126

The FCMSE algorithm is an algorithm that measures the complexity of a time

127 series as the sample entropy of the time series at different scales, which compensates

128 for the shortcomings of the MSE and CMSE algorithms (Kang et al. 2021). The main 129 steps are as follows:

(1)For the rainfall time series $x=\left\{x_{1}, x_{2}, \mathrm{~L}, x_{n}\right\}$, its $\tau$-th scale coarse-grained

131 process is

$$
y_{k, j}^{(\tau)}=\frac{1}{\tau} \sum_{i=(j-1) \tau+k}^{j \tau+k-1} x_{i}, 1 \leq j \leq \frac{n}{\tau}, 1 \leq k \leq \tau
$$

134 graining described above.

(2) Under the scale factor $\tau$, the number of all $\tau$ coarse-grained sequence

136 matching vector pairs $n_{k, \tau}^{m+1}$ and pairs $n_{k, \tau}^{m}$ is calculated.

(3) Calculate the mean $\overline{n_{k, \tau}^{m+1}}$ and the value $\overline{n_{k, \tau}^{m}}$ of $\tau$ the $n_{k, \tau}^{m+1}$ and $n_{k, \tau}^{m}$ at $1381 \leq k \leq \tau$. Define the FCMSE under the scale factor B as the logarithm of the ratio 139 of $\mathrm{N}$ and the value $\mathrm{M}$, i.e. 


$$
\operatorname{RCMSE}(x, \tau, m, r)=-\operatorname{In} \frac{\overline{n_{k, \tau}^{m+1}}}{\overline{n_{k, \tau}^{m}}}
$$

142 all $n_{k, \tau}^{m+1}$ and $n_{k, \tau}^{m}$ are zero.

\section{$143 \quad 1.3$ Stacking Algorithms}

144 In the Stacking Integrated Learning Model, the predictive power of each base

145 learner is analysed individually, while the combined effect of each base learner is

146 compared, so that the Stacking Integrated Learning Model achieves the best prediction

147 results (Shi et al. 2019). The base learner has to be selected for good performance but

148 with different model principles. The K-Nearest Neighbor algorithm is a well-theorized

149 classical machine learning method that is simple to implement and efficient to train ( $\mathrm{Lu}$

150 et al. 2021). Random Forest is one of the first machine learning algorithms that

151 improves the prediction accuracy of various models by constructing different training

152 sets to improve the variance of each classification model (Dong et al. 2020, Xiao et al

153 2020). Support vector regression shows good fitting performance for non-linear

154 regression prediction of high-dimensional, complex data (Xiong et al. 2006).Better

155 generalization and fitting performance of artificial neural network models (Gorai et

156 al.2021). In summary, this paper selects KNN, RF, SVR and ANN as the base learners,

157 and the ANN algorithm with strong generalization ability is used as the meta-learner.

\section{2 CEEMDAN-RCMSE-Stacking Model construction}

159 Based on the above-mentioned CEEMD algorithm, fine composite multiscale

160 entropy and Stacking algorithm, a short-term precipitation prediction model is built in

161 this paper, as shown in Figure 1. The specific ideas are as follows. 
(1) The raw precipitation data were decomposed into s modal components and 1

163 residual component using the CEEMD algorithm.

164 (2) The RCMSE values of each component are calculated and sequences with

165 similar entropy values are superimposed to form a new sequence of components.

166 (3) The reconstructed component data were divided into a training set D and a test

167 set $\mathrm{T}$.

168 (4) Using 5-fold cross-validation, the training set D is randomly divided into 5

169 equal parts. Let each base learner train 1 copy of the training set and use the remaining

1704 copies as the test set for prediction. Combine the predictions of the 4 base learners

171 into the training set $B^{c}$ of the meta-learner.

172 (5) Let each base learner make predictions on the test set $\mathrm{T}$. The average of the

173 predictions is used as the test set $f /$ for the meta-learner.

174 (6) A meta-learner is used to train the new training set $B^{C}$, and this meta-learner is

175 used to predict the new test set $t /$. The final prediction is output. 


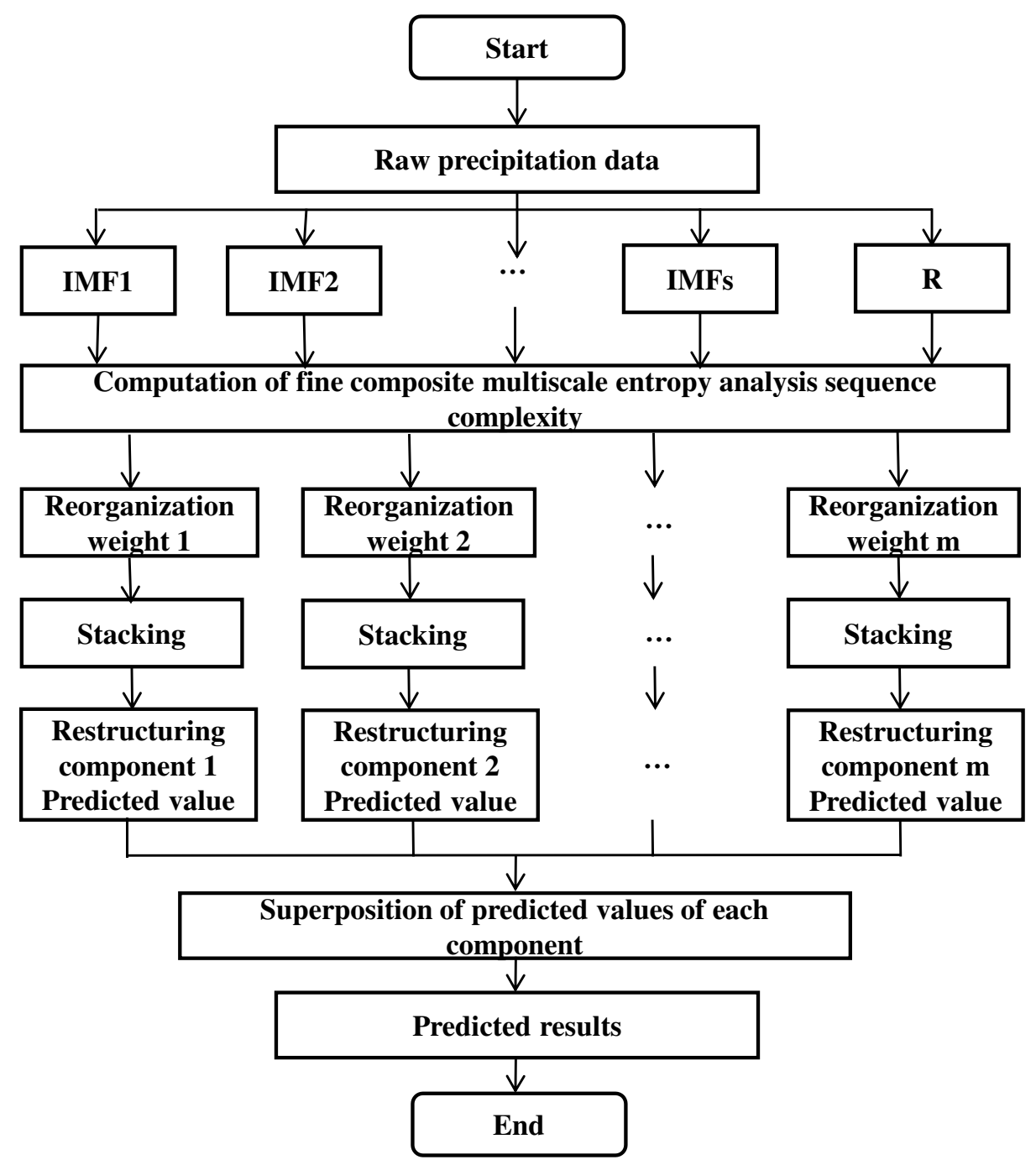

Fig1 Flow of CEEMDAN-RCMSE-Stacking based prediction model

\section{$178 \quad 3$ Example applications}

The data used in this paper are from actual precipitation measurements in the

180 Western Gorge, as shown in Figure 2, sampled from 1960 to 2019. A total of 720 data

181 points were used, with the first 660 data points used for the training set and the last 60

182 data points for the test set. In this paper, root mean square error (RMSE), mean absolute

183 error (MAE) and goodness-of-fit coefficient $R^{2}$ are used to evaluate the model error. 


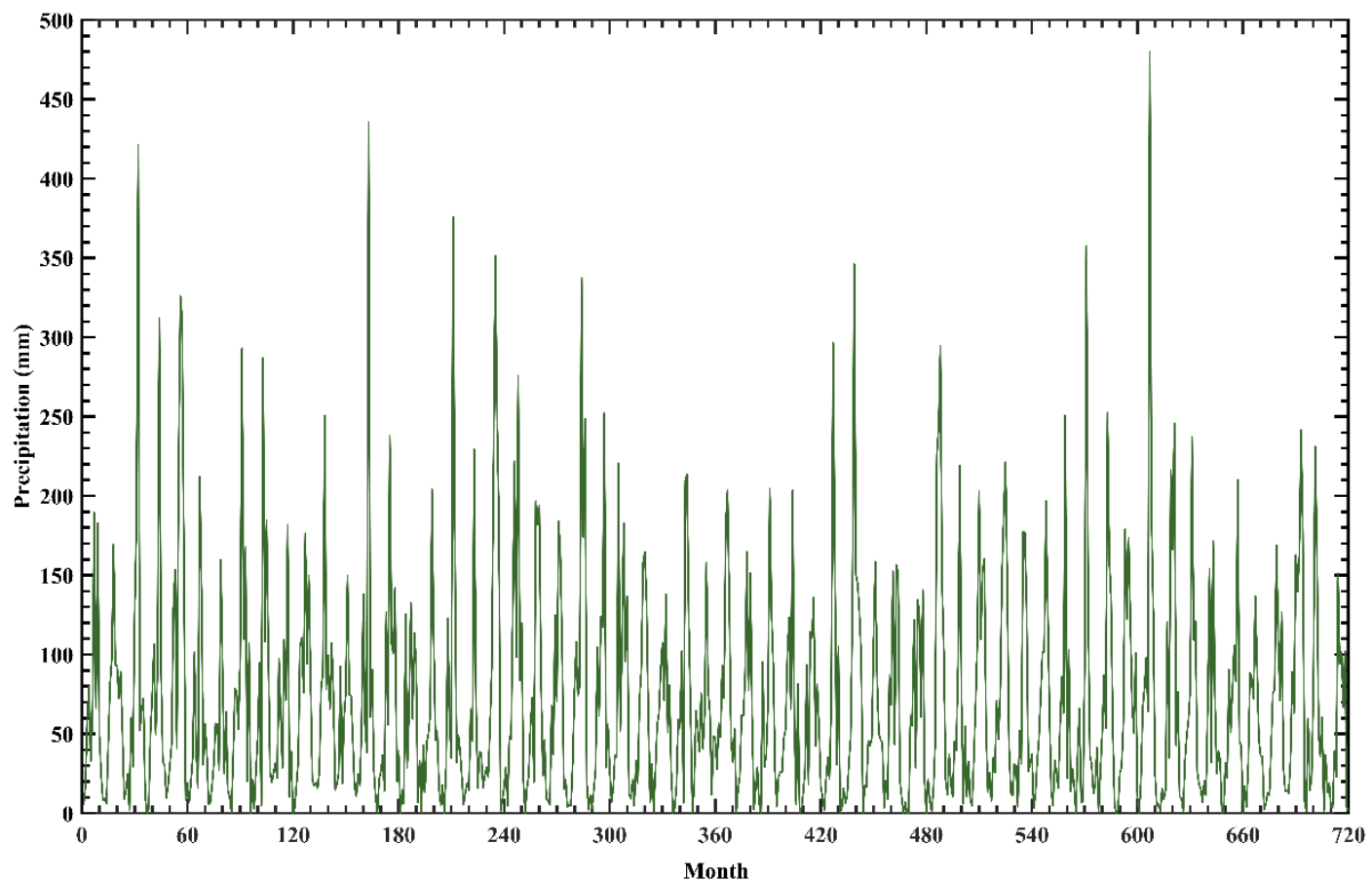

185

186

187

188

189

190 deviation $N_{\text {std }}=0.05$, and the maximum number of iterations MaxIter $=100$. The

191 decomposition results are shown in Figure3. 

deviation of component sequence time lag tau $=1$. FCMSE values for each IMF component and residual are listed in Table 1.

As can be seen from Figure 3, the precipitation series is decomposed by CEEMD into 7 modal components and 1 residual component at different frequency scales. Due to the non-smoothness of the series makes the decomposition result in a large number of component series, if the 5-fold method is used to model the prediction of each component directly it will lead to a sharp increase in computational effort. This paper therefore uses the RCMSE method to calculate the fine composite multiscale entropy values of each component in order to assess the complexity of each component, and based on this, the components are combined and reorganised. RCMSE test parameters: embedding dimension $m=2$, conditional threshold $\mathrm{r}$ takes 0.2 times standard

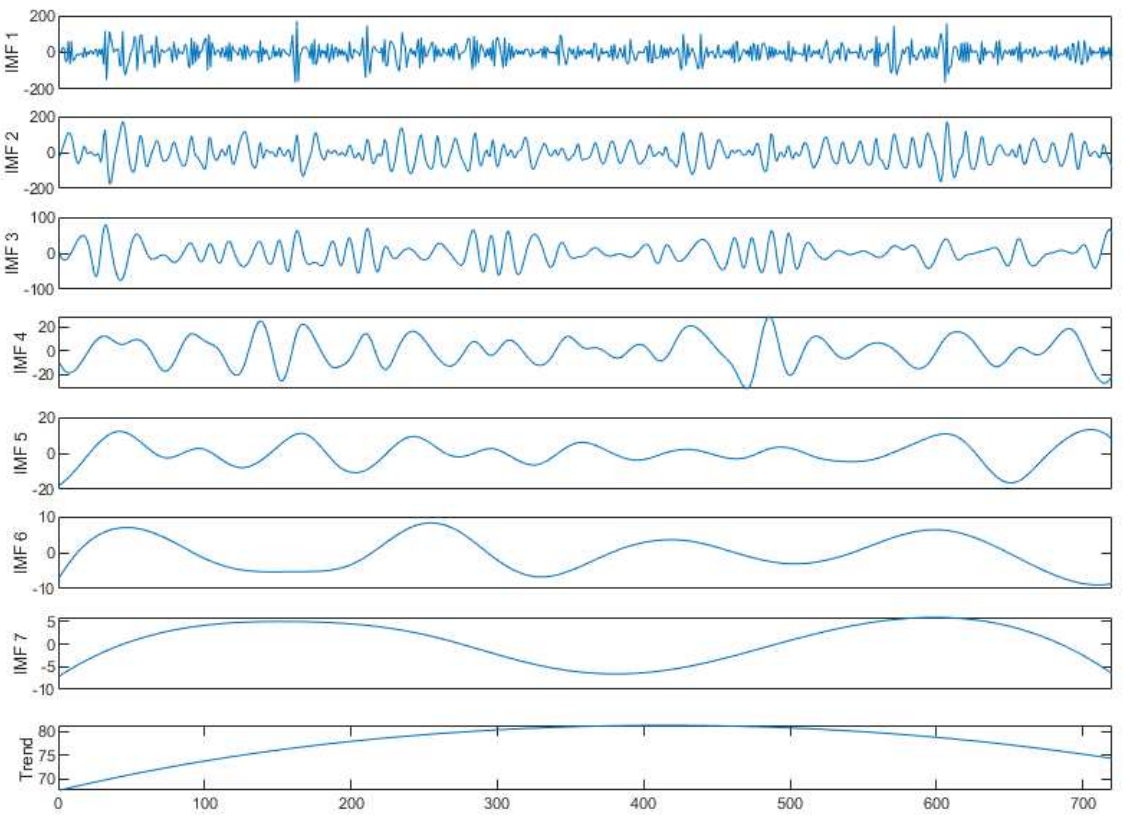

Fig3 CEEMD decomposition of monthly precipitation series results 205

Table 1 FCMSE values for IMF components and residuals

\begin{tabular}{cccc}
\hline Component sequences & FCMSE values & Component sequences & FCMSE values \\
\hline IMF1 & 0.1605 & IMF5 & 0.0443 \\
IMF2 & 0.1044 & IMF6 & 0.0243 \\
IMF3 & 0.0639 & IMF7 & 0.0089 \\
\hline
\end{tabular}




\begin{tabular}{cccc}
\hline IMF4 & 0.0560 & $\mathrm{R}$ & 0.0023 \\
\hline
\end{tabular}

As can be seen from Table 1, the RCMSE values for each component series are

207 mainly distributed in the four numerical neighbourhoods of $0.15,0.1,0.05$ and 0.005 .

208 Accordingly, in this paper, the component sequences are recombined and the results are

209 as follows: $\mathrm{F} 1=\mathrm{IMF} 1, \mathrm{~F} 2=\mathrm{IMF} 2, \mathrm{~F} 3=\mathrm{IMF} 3+\mathrm{IMF} 4+\mathrm{IMF} 5+\mathrm{IMF} 6$ and $\mathrm{F} 4=\mathrm{IMF} 7+\mathrm{R}$ are

210 the recombined components. Figure 4 shows the rainfall recombination component

211 sequences.
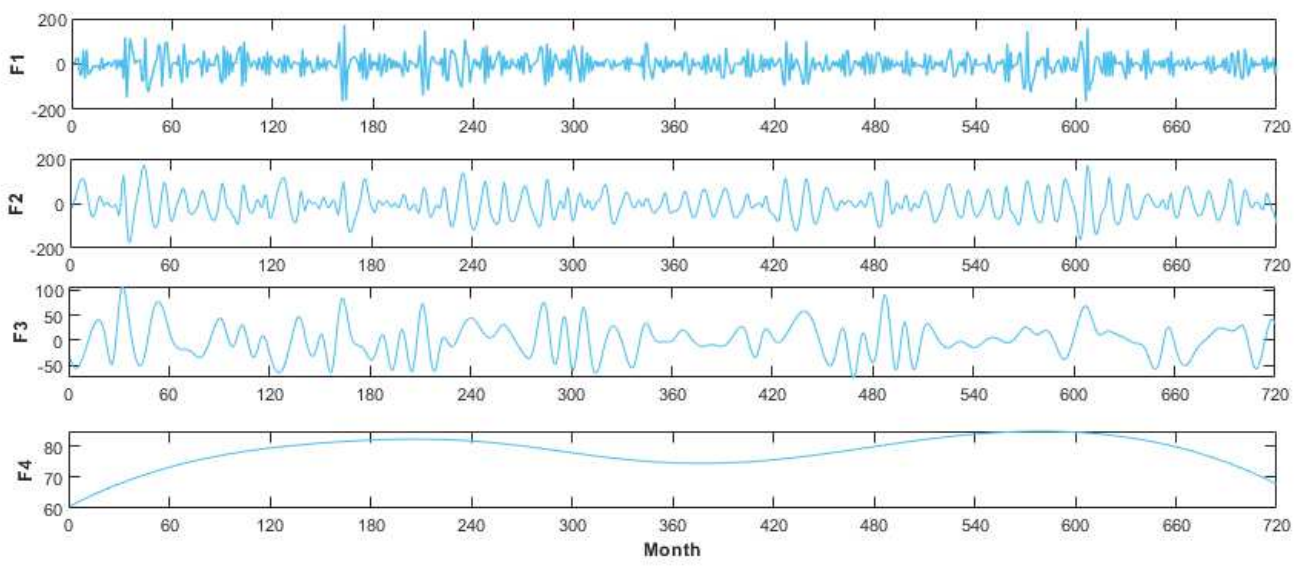

Fig4 FCMSE recombination component sequence results

The reorganised four components were imported into the Stacking model for

215 prediction and the results are shown in Figure 5. 

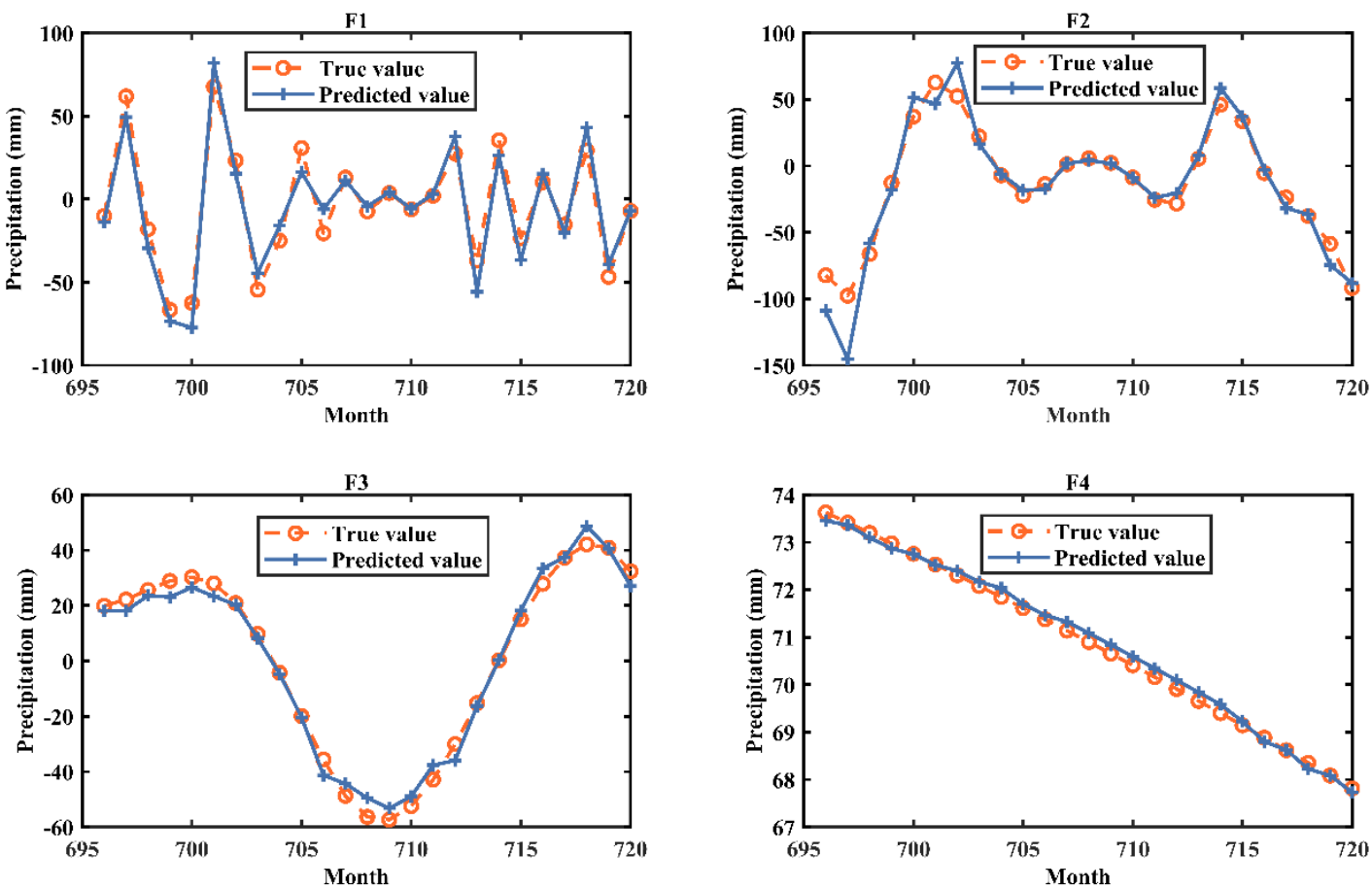

Fig5 F1-F4 prediction results

As can be seen from Figure 5, the smoothness of the Xixia precipitation time series

219 is enhanced and the volatility is significantly reduced after the CEEMD decomposition.

220 The prediction errors from F1 to F4 are getting lower and lower, which indicates that

221 the training is getting better and better.

\subsection{Cross-sectional comparison test of Stacking model precipitation prediction}

In order to compare the prediction accuracy of the Stacking prediction model, this

224 paper will use four base learners to do a cross-sectional comparison test of the

225 prediction of modal $\mathrm{F} 3$, taking into account the moderate frequency fluctuation and

226 smoothness of modal F3, which is more likely to reflect the fitting effect differently.

227 The values of each model fit error evaluation metric and precipitation prediction plots

228 for the F3 modal are shown in Figure 6 and listed in Table 2. 


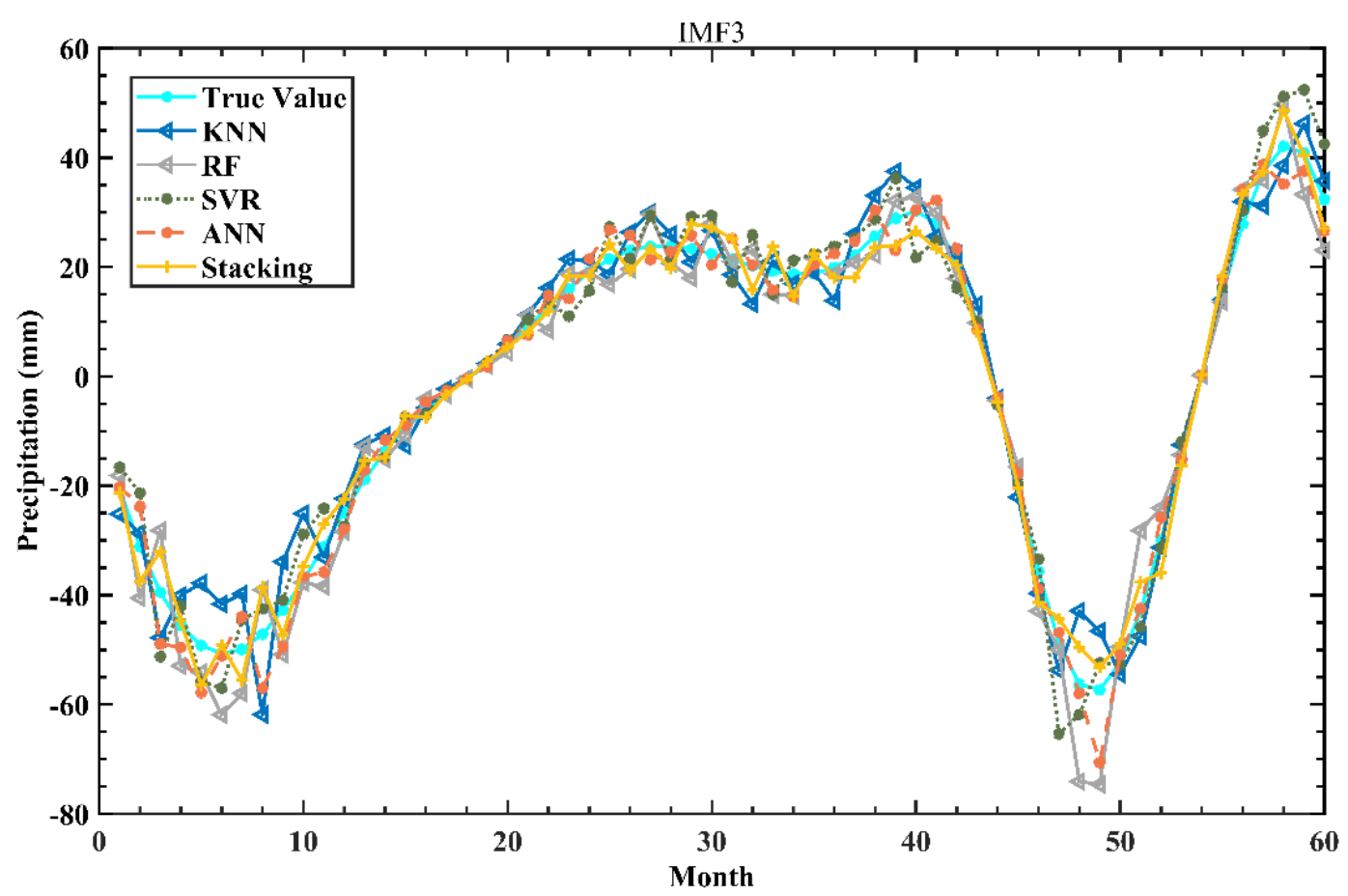

Fig6 Fitting of each model for the F3 mode

Table 2 Relative errors in the prediction results for the $\mathrm{F} 3$ trend term

\begin{tabular}{cccc}
\hline Predictive models & RMSE & MAE & $\mathrm{R}^{2}$ \\
\hline KNN & 26.78 & 20.39 & 0.8158 \\
RF & 26.04 & 19.17 & 0.8227 \\
SVR & 26.42 & 19.24 & 0.8213 \\
ANN & 25.14 & 18.16 & 0.8432 \\
Stacking & 22.52 & 15.10 & 0.8591 \\
\hline
\end{tabular}

It can be seen from Table 2 that in the base learner, the RMSE and MAE of the

233 ANN algorithm are the smallest, which are 25.14 and 18.16, respectively. From the

234 fitting effect, ANN model fitting degree is the highest. This indicates that the model

235 performs best and generalises best, and empirically demonstrates the rationality and

236 validity of setting the meta-learner of the Stacking model as an ANN model in this paper.

237 The Stacking model, which integrates four models, achieves better prediction

238 performance than the single model, with prediction errors RMSE and MAE $10.42 \%$ and

$23916.85 \%$ lower respectively than the ANN model, which is the best performing of the 240 single models. This is $15.91 \%$ and $25.94 \%$ lower than the KNN model, which is the 
241 worst performing of the single models, and the fit to the actual values of the modal F3

242 is $5.82 \%$ better.

\subsection{Five-year rainfall prediction test}

To further verify the generalisability and accuracy of the CEEMDAN-RCMSE-

245 Stacking model proposed in this paper, a total of 720 data points were selected from the 246 five-year 12-month precipitation data of the hydrological station, with the first 55 years 247 (660 data points) as the test set and the remaining 60 data points as the test set. The 248 comparison model is the LSTM model, a deep learning algorithm widely used in natural 249 language processing (Liu et al. 2020). The results of 60 months of wind power 250 predictions under each prediction model are shown in Table 4.

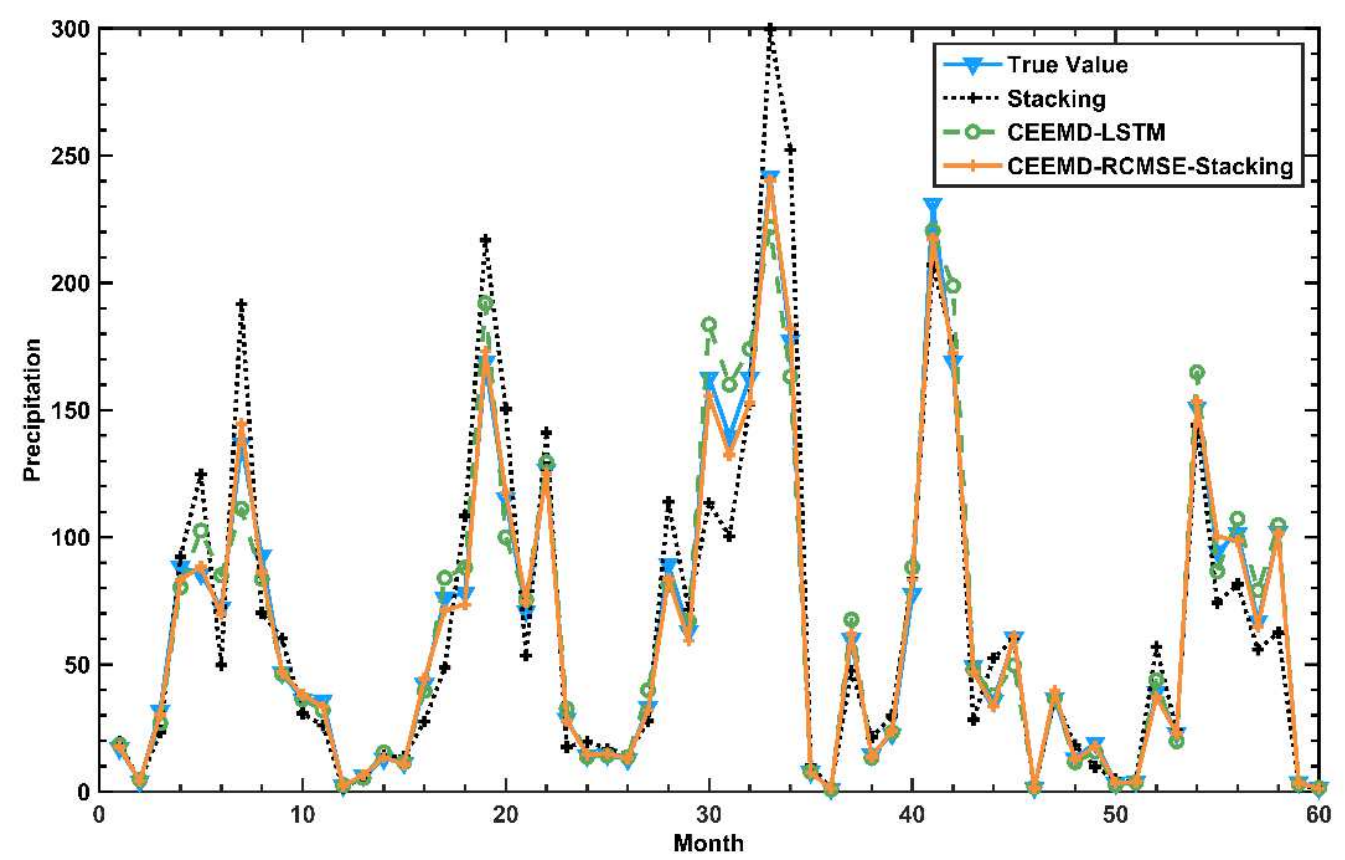

Fig7 Prediction results of the CEEMD-RCMSE-Stacking model compared with other models

As can be seen from Figure 7, the single Stacking model has a large error between

254 the predicted and measured values, and the fit is the worst, with the predictions obtained using the CEEMD-LSTM decomposition method deviating significantly from the peak 
257 best fit and the prediction results are closer to the actual rainfall, verifying the accuracy 258 and superiority of the model proposed in this paper.

Table 3 Comparison of prediction results from different models

\begin{tabular}{|c|c|c|c|c|c|c|c|}
\hline \multirow[b]{2}{*}{ Month } & \multirow{2}{*}{$\begin{array}{l}\text { True } \\
\text { value }\end{array}$} & \multicolumn{2}{|c|}{ Stacking } & \multicolumn{2}{|c|}{ CEEMD-LSTM } & \multicolumn{2}{|c|}{ CEEMD-RCMSE-Stacking } \\
\hline & & $\begin{array}{l}\text { Predicted } \\
\text { value }\end{array}$ & $\begin{array}{c}\text { Relative } \\
\text { error \% }\end{array}$ & $\begin{array}{c}\text { Predicted } \\
\text { value }\end{array}$ & $\begin{array}{c}\text { Relative } \\
\text { error \% }\end{array}$ & $\begin{array}{l}\text { Predicted } \\
\text { value }\end{array}$ & $\begin{array}{c}\text { Relative } \\
\text { error } \%\end{array}$ \\
\hline 1 & 17 & 19.77 & 16.28 & 18.53 & 9.00 & 17.53 & 3.14 \\
\hline 2 & 4 & 3.74 & 6.62 & 4.74 & 18.49 & 4.13 & 3.36 \\
\hline 3 & 31.7 & 23.38 & 26.25 & 26.91 & 15.11 & 30.32 & 4.36 \\
\hline 4 & 88.4 & 92.39 & 4.52 & 80.42 & 9.03 & 83.21 & 5.87 \\
\hline 5 & 85.6 & 124.82 & 45.82 & 102.59 & 19.85 & 88.51 & 3.40 \\
\hline 6 & 72.2 & 49.93 & 30.84 & 85.07 & 17.82 & 69.71 & 3.45 \\
\hline 7 & 136.9 & 191.60 & 39.96 & 111.25 & 18.74 & 144.43 & 5.50 \\
\hline 8 & 92.6 & 70.28 & 24.10 & 83.53 & 9.80 & 86.34 & 6.76 \\
\hline 9 & 46.7 & 60.23 & 28.97 & 46.27 & 0.93 & 46.87 & 0.37 \\
\hline 10 & 36.2 & 30.82 & 14.85 & 36.25 & 0.14 & 38.03 & 5.04 \\
\hline 11 & 35.9 & 26.06 & 27.40 & 32.01 & 10.84 & 33.46 & 6.80 \\
\hline 12 & 2.4 & 1.61 & 33.02 & 2.59 & 7.93 & 2.50 & 3.99 \\
\hline 13 & 6.2 & 5.86 & 5.43 & 5.36 & 13.55 & 6.53 & 5.25 \\
\hline 14 & 13 & 14.17 & 8.97 & 15.47 & 19.03 & 13.52 & 3.97 \\
\hline 15 & 11.3 & 13.65 & 20.82 & 11.60 & 2.69 & 10.86 & 3.88 \\
\hline 16 & 42.3 & 27.61 & 34.74 & 39.64 & 6.29 & 44.36 & 4.87 \\
\hline 17 & 76 & 48.78 & 35.82 & 84.05 & 10.59 & 71.39 & 6.06 \\
\hline 18 & 78.2 & 108.46 & 38.69 & 88.10 & 12.66 & 73.53 & 5.98 \\
\hline 19 & 168.8 & 216.88 & 28.48 & 192.01 & 13.75 & 173.04 & 2.51 \\
\hline 20 & 115.3 & 150.39 & 30.44 & 100.02 & 13.25 & 117.44 & 1.85 \\
\hline 21 & 70.9 & 53.51 & 24.53 & 75.49 & 6.47 & 74.81 & 5.51 \\
\hline 22 & 126.6 & 141.01 & 11.39 & 129.47 & 2.27 & 125.12 & 1.17 \\
\hline 23 & 28.6 & 17.69 & 38.15 & 32.66 & 14.21 & 27.97 & 2.21 \\
\hline 24 & 14.1 & 19.69 & 39.61 & 13.68 & 2.98 & 14.55 & 3.16 \\
\hline 25 & 14.5 & 16.92 & 16.66 & 14.20 & 2.05 & 14.92 & 2.87 \\
\hline 26 & 12.7 & 14.32 & 12.76 & 13.62 & 7.25 & 13.16 & 3.64 \\
\hline 27 & 33.3 & 27.87 & 16.30 & 39.93 & 19.91 & 31.94 & 4.09 \\
\hline 28 & 89.3 & 113.91 & 27.56 & 81.31 & 8.95 & 83.44 & 6.56 \\
\hline 29 & 63.1 & 71.58 & 13.44 & 67.13 & 6.38 & 59.36 & 5.92 \\
\hline 30 & 162.8 & 113.53 & 30.26 & 183.63 & 12.79 & 155.61 & 4.42 \\
\hline 31 & 139.7 & 100.42 & 28.12 & 159.97 & 14.51 & 132.41 & 5.22 \\
\hline 32 & 162.7 & 152.36 & 6.36 & 174.01 & 6.95 & 152.82 & 6.07 \\
\hline 33 & 241.7 & 299.75 & 24.02 & 221.76 & 8.25 & 240.16 & 0.64 \\
\hline 34 & 177.3 & 252.15 & 42.22 & 163.15 & 7.98 & 181.99 & 2.64 \\
\hline 35 & 7.8 & 10.42 & 33.59 & 7.88 & 0.97 & 7.61 & 2.47 \\
\hline 36 & 1.2 & 1.30 & 8.23 & 0.97 & 19.45 & 1.18 & 1.84 \\
\hline
\end{tabular}




\begin{tabular}{|c|c|c|c|c|c|c|c|}
\hline 37 & 59.9 & 47.65 & 20.45 & 67.63 & 12.91 & 62.06 & 3.61 \\
\hline 38 & 14.5 & 21.50 & 48.28 & 13.33 & 8.06 & 13.65 & 5.83 \\
\hline 39 & 22.6 & 29.79 & 31.81 & 23.36 & 3.35 & 24.03 & 6.35 \\
\hline 40 & 77.7 & 83.76 & 7.80 & 88.18 & 13.49 & 83.22 & 7.10 \\
\hline 41 & 231.1 & 207.00 & 10.43 & 220.46 & 4.60 & 217.30 & 5.97 \\
\hline 42 & 168.9 & 176.71 & 4.62 & 198.80 & 17.70 & 172.25 & 1.99 \\
\hline 43 & 49.5 & 28.33 & 42.77 & 48.08 & 2.88 & 47.03 & 5.00 \\
\hline 44 & 35.6 & 52.45 & 47.33 & 37.95 & 6.59 & 33.52 & 5.84 \\
\hline 45 & 60.4 & 60.52 & 0.20 & 49.71 & 17.70 & 61.32 & 1.52 \\
\hline 46 & 1.6 & 0.97 & 39.28 & 1.84 & 15.19 & 1.57 & 2.11 \\
\hline 47 & 36.8 & 36.94 & 0.38 & 36.66 & 0.38 & 39.47 & 7.26 \\
\hline 48 & 13 & 18.42 & 41.71 & 11.64 & 10.49 & 12.69 & 2.41 \\
\hline 49 & 19.3 & 9.80 & 49.25 & 15.83 & 17.99 & 17.92 & 7.16 \\
\hline 50 & 3.3 & 4.82 & 45.94 & 2.67 & 19.08 & 3.47 & 5.03 \\
\hline 51 & 3.9 & 3.79 & 2.84 & 3.68 & 5.75 & 3.80 & 2.58 \\
\hline 52 & 39.1 & 57.01 & 45.80 & 43.80 & 12.03 & 37.17 & 4.93 \\
\hline 53 & 23 & 24.04 & 4.54 & 19.85 & 13.69 & 23.06 & 0.24 \\
\hline 54 & 151.1 & 144.01 & 4.69 & 164.85 & 9.10 & 153.33 & 1.48 \\
\hline 55 & 94.5 & 74.20 & 21.48 & 86.60 & 8.36 & 100.26 & 6.10 \\
\hline 56 & 101.6 & 81.47 & 19.81 & 107.33 & 5.64 & 98.63 & 2.93 \\
\hline 57 & 67 & 55.83 & 16.66 & 79.18 & 18.17 & 65.06 & 2.90 \\
\hline 58 & 102 & 62.49 & 38.74 & 104.78 & 2.73 & 101.48 & 0.51 \\
\hline 59 & 3.7 & 2.38 & 35.60 & 3.17 & 14.40 & 3.45 & 6.67 \\
\hline 60 & 1.4 & 1.43 & 2.07 & 1.49 & 6.58 & 1.35 & 3.33 \\
\hline Aver & $\begin{array}{l}\text { lative } \\
0\end{array}$ & \multicolumn{2}{|c|}{24.29} & \multicolumn{2}{|c|}{10.30} & \multicolumn{2}{|c|}{4.06} \\
\hline \multicolumn{2}{|c|}{ RMSE } & \multicolumn{2}{|c|}{22.52} & \multicolumn{2}{|c|}{9.81} & \multicolumn{2}{|c|}{3.72} \\
\hline \multicolumn{2}{|c|}{ MAE } & \multicolumn{2}{|c|}{15.10} & \multicolumn{2}{|c|}{6.63} & \multicolumn{2}{|c|}{2.53} \\
\hline \multicolumn{2}{|c|}{ R2 } & \multicolumn{2}{|c|}{0.8591} & \multicolumn{2}{|c|}{0.9732} & \multicolumn{2}{|c|}{0.9960} \\
\hline
\end{tabular}

260 The statistical results of the evaluation criteria in Table 3 show that the combined

261 model CEEMD-FCMSE-Stacking is much better than the single Stacking forecasting

262 model, with a $15.94 \%$ improvement in the coefficient of goodness of fit $\mathrm{R}^{2}$ and $83.48 \%$

263 and $83.25 \%$ reductions in root mean square error RMSE and mean absolute error MAPE,

264 respectively. The CEEMD-LSTM also achieves a certain level of fit, but not as good as

265 the CEEMD-FCMSE-Stacking model at the peak points. Compared with the CEEMD-

266 LSTM model, the combined CEEMD-RCMSE-Stacking model improved the 267 goodness-of-fit coefficient R2 by $2.34 \%$, and reduced the root mean square error RMSE 
and mean absolute error MAPE by $62.08 \%$ and $61.84 \%$.

To improve the accuracy of medium- and long-term precipitation forecasts, this paper investigates the practicality and feasibility of the Stacking integrated learning model for runoff time series, constructs the CEEMD-FCMSE-Stacking prediction model, and verifies and compares the prediction effects of different models with the monthly precipitation data of the Xixia. The model performs well in precipitation forecasting tasks and is suitable for short-term precipitation forecasting with large seasonal fluctuations and for developing flood and drought plans, and can be used effectively for time series analysis in hydrology and related fields to mitigate the risk of climate extremes.

\section{Conclusion}

(1) To address the characteristics of intermittent and fluctuating precipitation, this paper introduces the CEEMD algorithm to decompose the precipitation series, which effectively reduces the non-smooth characteristics of the original series. At the same time, the CEEMD algorithm overcomes the possible modal confounding problem in EMD and provides a good basis for the Stacking model to make predictions. At the same time, the complexity of the CEEMD decomposition components is calculated using fine composite multiscale entropy, and they are restructured by FCMSE values, reducing the model complexity and computational scale.

(2) The CEEMD-FCMSE-Stacking model is a better fit than the CEEMD-LSTM coupled neural network model for the location of abrupt changes in precipitation data, and is more reasonably detailed in terms of reflecting the true variability of the series. 
290 The mean relative error of $4.06 \%$, RMSE and MAE of 3.72 and 2.53 respectively, are

291 both low, and the coefficient of goodness of fit $\mathrm{R}^{2}$ of 0.9960 is very close to 1 . The

292 prediction accuracy is better than that of the CEEMD-LSTM model. The empirical

293 results show that the model overcomes the limitations of the coupled model and

294 substantially improves the generalization ability and accuracy of the precipitation 295 prediction.

296 (3) Although the overall prediction fitting degree of the established CEEMD297 RCMSE-Stacking model is relatively high, RMSE and MAE are relatively large. When 298 selecting the base learner and meta-learning, conservative methods are used to select 299 the simple learning methods that are common in recent years. There is no bold choice 300 that has not been applied to the precipitation prediction method. In the subsequent work, 301 further research will be carried out to improve the accuracy of the Stacking integrated 302 learning model.

303 Availability of data and materials Data and materials are available from the 304 corresponding author upon request.

305 Author contribution All authors contributed to the study conception and design. 306 writing and editing: Xianqi Zhang and Kai Wang; chart editing: Tao Wang; preliminary 307 data collection: Kai Wang. All authors read and approved the final manuscript.

308 Funding This work was supported by the Key Scientific Research Project of Colleges 309 and Universities in Henan Province (CN) [grant numbers 17A570004].

\section{Declarations}

311 Ethical approval and consent to participate Not applicable.

312 Consent to publish Written informed consent for publication was obtained from all 313 participants. 
314 Competing interests The authors declare no competing interests

315

\section{References}

Bidroha B, Maria N, Alan O C.(2020) New Approach to Multisite Downscaling of Precipitation by Identifying Different Set of Atmospheric Predictor Variables[J]. Journal of Hydrologic Engineering,2020,25(5):

Dong N, Ke K W, Li J S. (2020) Short-term photovoltaic power generation forecasting based on random forest feature selection and CEEMD: A case study[J]. Applied Soft Computing Journal, 93:

Fethi O, Mourad L, Soltane A. (2018) Improvement of rainfall estimation from MSG data using Random Forests classification and regression[J]. Atmospheric Research, 211:

Guo J, Guo S L, Chen H. (2010). ANN statistical downscaling method for predicting precipitation changes in the Han River basin[J]. Journal of Wuhan University (Engineering Edition), 43(02):148-152.

Gorai A K, Himanshu V K, Santi C. (2021)Development of ANN-Based Universal Predictor for Prediction of Blast-Induced Vibration Indicators and its Performance Comparison with Existing Empirical Models[J]. Mining, Metallurgy \& Exploration: Kang W H, Xu T Q, Wang Y G. (2021) Short-term wind power forecasting based on CEEMDAN-fine composite multiscale entropy and Stacking integrated learning $[\mathrm{J} / \mathrm{OL}]$. Water Resources and Hydropower Technology (in Chinese and English):1-13. 
336 Indian summer monsoon rainfall forecasting $[\mathrm{J}]$. Theoretical and Applied Climatology: Khan Md. Munir H, Muhammad N S, (2020). Wavelet based hybrid ANN-ARIMA

338 models for meteorological drought forecasting[J]. Journal of Hydrology, 590:

Risk Framework for Urban Tourism under Two Rainfall Scenarios[J]. Sustainability, 341 13(5):

Lu K, Wu W, Lin G R. (2021) Combined prediction method of passenger hub aggregation based on KNN regression[J]. Journal of Jilin University (Engineering Edition),51(04):1241-1250.

Liu X, Zhao N, Guo JY. (2020) Monthly precipitation prediction on the Tibetan

346 Plateau based on LSTM neural network[J]. Journal of Geoinformation Science, 22(08):1617-1629.

348 Lin, Y G, Du Y, Meng Y. (2021) Study on the influence of different integrated 349 models on short time runoff forecasting in small watersheds[J/OL]. China Rural Water 350 Conservancy and Hydropower:1-15. Li Y W. (2021) Research and application of runoff time series prediction based on 352 integrated learning[D]. University of Electronic Science and Technology. Shi J Q, Zhang J H. (2019) Load forecasting method based on multi-model fusion 357 Stacking integrated learning approach[J]. Chinese Journal of Electrical Engineering, 
39(14):4032-4042.

Song Y T, Zhao X H, Zhu X P, Xi D J. (2019) Monthly runoff prediction of the

360 upper Fen River based on CEEMD-BP model[J]. People's Yellow River, 41(08):1-5.

361 Sun A, Li J Z, Feng P. (2021). Applicability of RF-SVR downscaling model in the

362 Luan River basin[J]. Journal of Water Resources and Water Engineering, 32(02):31-37.

363 Xiao S G, Mo L, Zhang X. (2020) Short-term load forecasting based on

364 CEEMDAN+RF+AdaBoost[J]. Hydropower Energy Science, ,38(04):181-184+175.

365 Xiong W L, Xu B G. (2006) Study on the optimal selection method of SVR

366 parameters based on PSO[J]. Journal of System Simulation, (09):2442-2445.

Yu X, Ling G, Li H H. (2018) A SVR - ANN combined model based on ensemble

368 EMD for rainfall prediction[J]. Applied Soft Computing Journal,2018: 